\title{
(C) OPEN ACCESS \\ Effects of injectable progestogen contraception versus the copper intrauterine device on HIV acquisition: sub-study of a pragmatic randomised controlled trial
}

\author{
G Justus Hofmeyr, ${ }^{1}$ Mandisa Singata-Madliki, ${ }^{1}$ Theresa A Lawrie, ${ }^{1,2}$ \\ Eduardo Bergel, ${ }^{4}$ Marleen Temmerman ${ }^{3}$
}

${ }^{1}$ Effective Care Research Unit, Eastern Cape Department of Health/Universities of the Witwatersrand, Walter Sisulu and Fort Hare, South Africa

${ }^{2}$ Royal United Hospital, Bath, UK ${ }^{3}$ Institute for Clinical Effectiveness and Health Policy (IECS), Buenos Aires, Argentina ${ }^{4}$ International Centre for Reproductive Health, Ghent University, Ghent, Belgium

\section{Correspondence to} Dr Theresa Lawrie, Cochrane Office, Education Centre, Royal United Hospital, Bath BA1 3NG, UK; tess@lawrie.com

Received 11 August 2016 Revised 21 November 2016 Accepted 16 January 2017 Published Online First 29 March 2017
CrossMark

To cite: Hofmeyr GJ, SingataMadliki M, Lawrie TA, et al. J Fam Plann Reprod Health Care 2017:43:175-180.

\section{ABSTRACT \\ Background Evidence from observational studies suggests an increased risk of HIV acquisition among women using depot medroxyprogesterone acetate (DMPA) contraception.}

Methods Within the context of a South African programme to increase women's access to the intrauterine contraceptive device (IUD), we conducted a pragmatic, open-label, parallel-arm, randomised controlled trial (RCT) of the IUD versus injectable progestogen contraception (IPC) at two South African hospitals. The primary outcome was pregnancy; secondary outcomes included HIV acquisition. Consenting women attending termination of pregnancy services were randomised after pregnancy termination between July 2009 and November 2012. Condoms were promoted for the prevention of sexually transmitted infections. Voluntary HIV testing was offered at baseline and at 12 or more months later. Findings on HIV acquisition are reported in this article.

Results HIV acquisition data were available for 1290 initially HIV-negative women who underwent a final study interview at a median of 20 months after randomisation to IPC or an IUD. Baseline group characteristics were comparable. In the IPC group, 545/656 (83\%) of participants received DMPA, 96 (15\%) received injectable norethisterone enanthate, $14(2 \%)$ received the IUD and one received oral contraception. In the IUD group 609 (96\%) received the IUD, 20 (3\%) received IPC and 5 (1\%) had missing data. According to intention-to-treat analysis, HIV acquisition occurred in 20/656 (3.0\%) women in the IPC arm and 22/634 (3.5\%) women in the IUD arm (IPC vs IUD, risk ratio 0.88; $95 \%$ confidence interval $0.48-1.59 ; p=0.7$ ).

\section{Key message points}

- The net physiological and behavioural effects of hormonal contraception on HIV acquisition cannot be predicted from existing animal models and observational clinical data.

- To date there is no evidence from randomised controlled trials (RCTs) which confirms a greater risk with injectable progestogens than with the copper intrauterine device.

- Larger RCTs are needed to determine the relative risks of HIV acquisition with various contraceptive methods.

- Contraception providers should continue to counsel women on the measures available to prevent HIV acquisition.

Conclusions This sub-study was underpowered to rule out moderate differences in HIV risk, but confirms the feasibility of randomised trial methodology to address this question. Larger $\mathrm{RCTs}$ are needed to determine the relative risks of various contraceptive methods on HIV acquisition with greater precision.

Trial registration number Pan African Clinical Trials Registry number PACTR201409000880157 (04-09-2014).

\section{BACKGROUND}

The possibility that hormonal contraception, particularly depot medroxyprogesterone acetate (DMPA), increases HIV 
acquisition has been of concern for almost three decades. DMPA increases the susceptibility of nonhuman primates to simian immunodeficiency virus (SIV) infection, ${ }^{1}{ }^{2}$ and studies in mice suggest that DMPA may increase mucosal permeability, promote tissue inflammation, and increase susceptibility to viral infection (herpes simplex type 2). ${ }^{3}$ In DMPA contraception users, mechanisms such as alterations in local and systemic immunity, cervicovaginal epithelial thinning or disruption, changes in vaginal flora, and increased risk of cervicovaginal infections might play a role in increasing HIV risk. ${ }^{4-6}$ However, a review of the biological link between HIV risk and hormonal contraception concluded that there is a paucity of rigorous research to support these potential mechanisms. $^{7}$

Following the conduct of an individual patient data meta-analysis derived from observational studies, ${ }^{8}$ which found the HIV acquisition risk with DMPA compared with no hormonal contraception [adjusted hazard ratio (aHR)] to be 1.22, (95\% confidence interval (95\% CI) 0.99-1.50; five studies), and another updated systematic review that estimates a HR of $1.4,{ }^{9}$ there remains considerable uncertainty about this potential association and the global public health implications. Observational studies are informative but have inherent methodological limitations as they are not designed to determine whether an association might be causal, and are unable to control for unknown confounders or exclude the possibility of a difference in inherent risk for HIV acquisition related to a woman's contraceptive choice. In addition, injectable progestogen (progestin) contraception (IPC) is a widely used and acceptable method of contraception in many low- and middle-income countries, and undermining its use as a contraceptive option without robust evidence of harm could have a substantial impact on unintended pregnancy and maternal mortality.

DMPA is the most widely used contraceptive method in South Africa, where the prevalence of HIV infection among women aged between 20 and 34 years old was $31.6 \%$ in $2012 .{ }^{10}$ In this article we report HIV acquisition data from a prespecified substudy of a pragmatic randomised controlled trial (RCT) of the copper intrauterine contraceptive device (IUD) versus IPC conducted within the context of a South African programme to increase women's access to the IUD. The primary article on pregnancy rates and method discontinuation has been published in BMC Reproductive Health. ${ }^{11}$ The sub-study objective was to determine whether IPC and the IUD differ in their effect on HIV acquisition.

\section{METHODS}

\section{Design and participants}

The trial was a pragmatic, open-label, parallel-arm RCT conducted at two Eastern Cape hospitals, Frere Hospital and Cecelia Makiwane Hospital, in South
Africa. Women attending termination of pregnancy services at the study sites who requested long-term contraception and met the inclusion criteria (i.e. intended to continue contraception for at least 1 year, were 16-45 years old, had no evidence of active pelvic infection on history and clinical examination, had no contraindications to IPC or IUD use, were prepared to use either method of contraception, understood the participant information form, and were willing to sign informed consent) were offered participation in the trial.

\section{Randomisation}

Between July 2009 and November 2012, consenting women were randomly allocated to an IUD or IPC as per the methods previously published. ${ }^{11}$ In brief, randomisation was performed according to a computergenerated random sequence in balanced blocks of variable size with a randomisation ratio of $1: 1$, and participants were allocated to study arms via sequentially numbered, sealed, opaque envelopes containing the group allocations once they had been entered onto the trial register. The study protocol identified the IUD as the experimental intervention as it had been recently introduced, and DMPA, the most commonly used method in our setting, as the comparator. For this HIV acquisition sub-study, results are presented with DMPA as the experimental arm for consistency with other studies in this field.

\section{Prespecified outcomes}

The primary outcome of the main study was pregnancy. Secondary outcomes were method discontinuation, side effects and HIV acquisition. Findings on HIV acquisition, evaluated in the subgroup of participants that were HIV-negative at baseline, are the focus of this article.

\section{Procedure}

Participants randomised to the IUD arm had their IUD inserted by the hospital staff after their termination of pregnancy, which was usually performed by manual vacuum aspiration. Similarly, in the IPC arm, the first injection was administered by hospital staff according to routine practice. In most instances DMPA was used. However, as this was a pragmatic trial within the routine services, allowance was made for the use of NET at the discretion of the provider. Women received no additional reminders to support continuation with the allocated contraception method.

Professional nurses working as research assistants recorded participant baseline details on a paper case report form and administered follow-up questionnaires by telephone at 3, 6, 9 and 12 months after randomisation. All women were offered counselling and voluntary HIV testing at baseline, according to national health policy, using rapid tests or laboratorybased ELISA tests. Those with positive results were 
offered a CD4 count and treatment according to national health guidelines.

At the 12-month telephone interview, participants were invited to attend a final interview at the study site and offered a follow-up HIV test, if appropriate. During the telephone interview, participants were asked the date and result of their most recent HIV test (and CD4 count, if positive), along with the name of the clinic where the result could be verified, and which method of contraception they were currently using, if any. For participants who did not attend the research site for a follow up HIV test at least 12 months after randomisation, the telephone report of their last result was used. Continued attempts were made to contact women until the close of the study, resulting in long follow-up times in some women.

\section{Sample size and analysis}

The sample size calculation was based on the primary outcome (pregnancy). As previously published, to detect a reduction in pregnancy rates from $2.5 \%$ to $1.5 \%$, we calculated that we needed 6546 participants $\left[\alpha=0.05, \beta=0.20\right.$; Epi Info $^{\mathrm{TM}} 7$ statistical software (Centres for Disease Control and Prevention)] and planned to recruit a total sample size of 7000 women to allow for loss to follow-up. Based on an HIV prevalence of $25 \%$, this would have allowed for a sample size for the sub-study of HIV-negative women of approximately 5250 (sufficient to detect an increase in HIV acquisition risk from $2 \%$ to $3.3 \%$ ). Sub-study data on HIV acquisition for IPC versus IUD arms were compared according to intention-to-treat (ITT) and per protocol analyses. We compared event rates as risk ratios (RR) with 95\% confidence intervals (95\% CIs). Values for $p$ were calculated in Epi Info 7 using the Chi-squared $\left(\chi^{2}\right)$ test with Mantel-Haenszel correction or, for small numbers, the Fisher's exact test. To check the robustness of the findings on HIV acquisition, we performed sensitivity analysis excluding women with self-reported HIV test results.

\section{Ethics, consent and permissions}

Ethical approval for the trial was obtained from the University of the Witwatersrand Committee for Research on Human Subjects, South Africa, on 25 April 2008, clearance certificate M080466. Signed informed consent to participate in the study was obtained from all participants. No payments or other incentives were offered, other than payment of transport costs for the final follow-up visit.

\section{Trial registration}

Prospective registration of the protocol with the South African National Clinical Trials Register was undertaken on 15 September 2008 (Verification Code: 0 -953). It was subsequently found not to have been logged by the system, and was re-registered with the Pan African Clinical Trials Registry on 4 September 2014 (PACTR201409000880157).

\section{RESULTS}

The trial closed early due to international concerns raised about the possible association between DMPA and HIV acquisition, following publication of an observational study reporting increased HIV acquisition among women who chose DMPA contraception, ${ }^{12}$ and the subsequent plan by international collaborators to conduct a large, multicentre trial to address the DMPA/HIV question, following a World Health Organization (WHO) Technical Consultation. ${ }^{13} 14$

At trial closure, 2493 (36\%) of the target sample had accrued, with 1246 and 1247 women randomised to the IPC and IUD arms, respectively. Some 475 (19\%) women tested HIV-positive at baseline, 14 $(0.6 \%)$ had missing test results, $243(10 \%)$ declined testing and $9(0.4 \%)$ had missing data on testing status. For the $1752(70.3 \%)$ women who tested HIV-negative at baseline (878 and 874 women in the IPC and IUD groups, respectively), and for those with follow-up data available, baseline characteristics of the two arms were similar (Figure 1 and Table 1).

Follow-up HIV test results, by investigatorperformed HIV test $(814 / 1290 ; 63 \%)$ or self-report $(476 / 1290 ; 37 \%)$, were available for $1290(74 \%)$ of these women. Thus, a total of 656 and 634 women had negative baseline HIV tests and subsequent HIV test results in the IPC and IUD study arms, respectively, and were included in the ITT analysis on HIV acquisition. Loss to follow-up for this outcome, due to loss of contact with participants or lack of a repeat HIV test, occurred with a similar frequency in the IPC $(25 \%)$ and IUD (27\%) study arms.

The time from enrolment to follow-up HIV test was similar for both study arms, with a median of 19.4 months in the IPC arm [interquartile range (IQR) 15.6-27.7] and 20.0 months (IQR 16.2-27.8) in the IUD arm. In the IPC group, 545/656 (83\%) of participants received DMPA, $96(15 \%)$ received injectable norethisterone enanthate (NET), 14 (2\%) received the IUD and one received oral contraception. In the IUD group, 609 (96\%) received the IUD, 20 (3\%) received IPC and 5 (1\%) had missing data. At follow-up, 553/656 (84\%) and 463/634 (73\%), respectively, reported currently using the allocated method. The incidence of HIV acquisition according to ITT analysis was 20/656 (3.0\%) in the IPC arm and 22/634 (3.5\%) in the IUD arm (IPC vs IUD, RR 0.88; 95\% CI 0.48-1.59; $p=0.7$ ) (Table 2).

For participants that had HIV tests performed by research staff, as opposed to those self-reporting HIV test results, the RR for HIV acquisition was 0.78 (95\% CI 0.38-1.60), with 13/411 (3.2\%) acquiring HIV during the study period in the IPC arm compared with 17/403 (4.1\%) in the IUD arm.

Per protocol analysis according to interventions received at baseline yielded similar results, with 21/ 661 (3.2\%) and 21/623 (3.4\%) acquiring HIV among those who received IPC and IUDs, respectively 


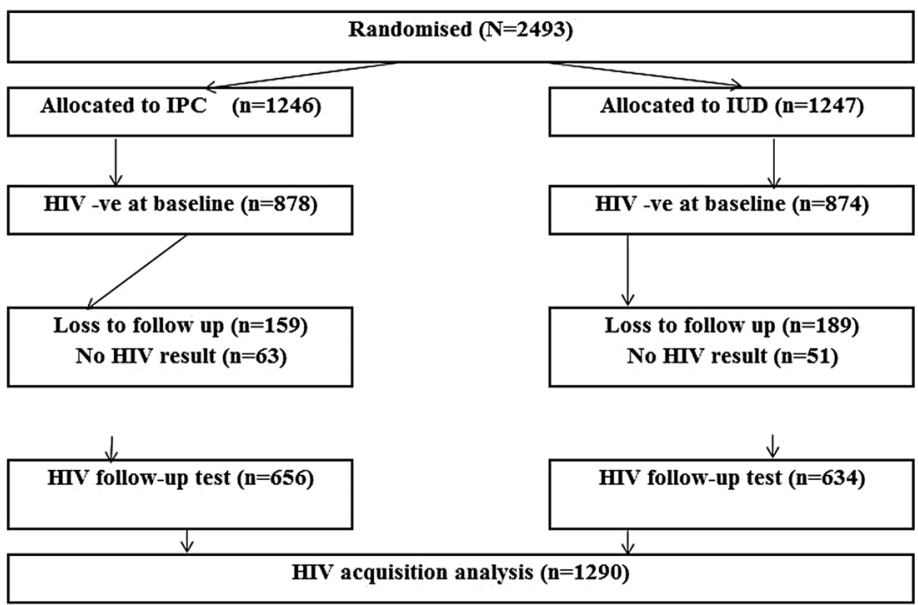

Figure 1 Flow of participants in sub-study analysis on HIV acquisition. IPC, injectable progestogen contraception; IUD, intrauterine device; -ve, negative.

Table 1 Baseline data of sub-study participants (HIV-negative at enrolment) expressed as numbers (\%) or mean values (SD]

\begin{tabular}{|c|c|c|c|c|c|c|c|c|}
\hline \multirow[b]{3}{*}{ Characteristic } & \multicolumn{4}{|c|}{ All participants with HIV-negative status at baseline } & \multicolumn{4}{|c|}{ Participants with follow-up } \\
\hline & \multicolumn{2}{|l|}{ IPC group $(N=878)$} & \multicolumn{2}{|l|}{ IUD group $(N=874)$} & \multicolumn{2}{|l|}{ IPC group $(N=656)$} & \multicolumn{2}{|l|}{ IUD group $(N=634)$} \\
\hline & $n(\%)$ or mean $[S D]$ & $N$ & $n(\%)$ or mean $[S D]$ & $N$ & $n(\%)$ or mean $[S D]$ & $N$ & $n(\%)$ or mean $[S D]$ & $N$ \\
\hline Age (years) & $25.7[6.2]$ & 873 & $26.0[6.3]$ & 867 & $26.0[6.3]$ & 655 & $26.0[6.3]$ & 631 \\
\hline Weight (kg) & $69.9[16.3]$ & 860 & $71.2[18.2]$ & 853 & $70.3[16.2]$ & 644 & 71.9 [18.2] & 615 \\
\hline Previous miscarriage & $76(8.7)$ & 878 & $85(9.7)$ & 874 & $66(10.1)$ & 656 & $68(10.7)$ & 634 \\
\hline Previous caesarean section & $98(11.2)$ & 878 & $112(12.8)$ & 874 & $72(11.0)$ & 656 & $76(12.0)$ & 634 \\
\hline Previous pelvic sepsis* & $162(18.5)$ & 878 & $170(19.5)$ & 874 & $120(18.3)$ & 656 & $170(19.5)$ & 634 \\
\hline Hypertension & $4 \quad(0.5)$ & 878 & $5 \quad(0.6)$ & 874 & $2(0.3)$ & 656 & $3(0.5)$ & 634 \\
\hline Diabetes mellitus & $1(0.1)$ & 878 & $3(0.3)$ & 874 & $1(0.1)$ & 656 & $3(0.5)$ & 634 \\
\hline \multicolumn{9}{|l|}{ Previous contraception use } \\
\hline IPC & $565(71.7)$ & 788 & $562(71.4)$ & 787 & $438 \quad(75)$ & 586 & $425 \quad(75)$ & 566 \\
\hline OC & $48 \quad(5.8)$ & 830 & $64 \quad(7.7)$ & 827 & $32(5.2)$ & 616 & $44 \quad(7.4)$ & 598 \\
\hline IUD & $1 \quad(0.1)$ & 830 & $1 \quad(0.1)$ & 827 & $1 \quad(0.1)$ & 616 & $1 \quad(0.1)$ & 598 \\
\hline
\end{tabular}

*South Africa implements the World Health Organization Primary Care model whereby women with certain signs and symptoms are treated 'syndromically' for assumed pelvic infection. This may result in over-diagnosis.

IPC, injectable progestogen contraception; IUD, intrauterine device; OC, oral contraception; SD, standard deviation.

$(p=0.8)$. Some $19 / 558(3.4 \%)$ women given DMPA at baseline and 2/103 (1.9\%) women given NET at baseline acquired HIV. Out of five women with missing data on the method received at baseline and one who received oral contraception, none acquired HIV.

During follow-up attempts, research assistants learned that two women in the sub-study had died; one women aged 18 years, who received DMPA, died from eclampsia 16 months after enrolment; the other women, aged 20 years, who received NET, died from an asthmatic attack 9 months after enrolment.

\section{DISCUSSION}

Our sub-study has several important limitations: The trial was terminated prematurely following a meeting of experts at the WHO headquarters in Geneva in 2012 that highlighted a perceived global need for a rigorous, well-funded and high-profile trial to answer the question regarding HIV acquisition. ${ }^{14}$ This global imperative did not exist at the time that our study was commenced. Early closure meant that the sample size for the sub-study did not have the power to exclude the possibility of a meaningful difference in effect on HIV acquisition between the two contraceptive methods tested. This limited its value to that of a pilot feasibility study. The contraceptive care and follow-up of the women took place within the routine health services, and was not under the supervision of the research team. The determination of HIV status at follow-up was based on rapid tests or laboratory-based ELISA tests, either performed by our research staff or self-reported by the women. In the latter case there may have been under-reporting. However, the women with self-reported tests provided the date and name of the clinic where the test was performed, and all selfreported results that could be confirmed by contacting 
Table 2 Results for HIV acquisition according to intention-totreat and per protocol analyses (initial method received)

\begin{tabular}{|c|c|c|c|c|c|}
\hline \multirow[b]{2}{*}{ Analysis } & \multicolumn{2}{|c|}{ HIV acquisition } & \multicolumn{2}{|c|}{$\begin{array}{l}\text { Effect estimate } \\
\text { (IPC vs IUD) }\end{array}$} & \multirow[b]{2}{*}{$p$} \\
\hline & $n(\%)$ & $N$ & $\mathbf{R} \mathbf{R}$ & $95 \% \mathrm{Cl}$ & \\
\hline \multicolumn{6}{|c|}{ ITT analysis (i.e. according to group allocation) } \\
\hline IPC & $20(3.0)$ & 656 & 0.88 & $0.48-1.59$ & 0.7 \\
\hline IUD & $22(3.5)$ & 634 & 1 & & \\
\hline \multicolumn{6}{|c|}{ PP analysis (i.e. according to initial method received) } \\
\hline$I P C$ & $21(3.2)$ & 661 & 0.94 & $0.52-1.71$ & 0.8 \\
\hline DMPA & $19(3.4)$ & 558 & 1.01 & $0.55-1.86$ & 1.0 \\
\hline NET & $2(1.9)$ & 103 & 0.58 & $0.14-2.42$ & 0.4 \\
\hline IUD & $21(3.4)$ & 623 & 1 & & \\
\hline OC & $0 \quad(0)$ & 1 & & & \\
\hline Missing data & $0 \quad(0)$ & 5 & & & \\
\hline
\end{tabular}

$\mathrm{Cl}$, confidence interval; DMPA, depot medroxyprogesterone acetate; IPC, injectable progestogen contraception; ITT, intention-to-treat; IUD, intrauterine contraceptive device; NET, norethisterone enanthate; $O C$, oral contraception; PP, per protocol; RR, risk ratio.

the relevant clinics were found to be correct. Any systematic bias due to under-reporting is likely to have affected both arms equally. In addition, the RR for HIV acquisition remained unchanged after exclusion of the self-reported results. We were not able to exclude 'window period' false-negative results at baseline or at follow-up, which would also be expected to affect both arms equally.

Within the IPC group, the characteristics of women who received NET may have differed from those who received DMPA; for this reason we have not drawn conclusions from the per protocol comparison of results in women who received NET or DMPA, which were reported for completeness. The ITT analysis retains the comparability of the arms as originally randomised [(DMPA plus NET) vs IUD]. If, for example, DMPA was associated with higher HIV acquisition risk than both NET and the IUD, use of NET by about $16 \%$ of women would have reduced the magnitude of the difference between IPC and IUD HIV acquisition risk but would not have eliminated it.

An important aspect of interpretation is that one cannot assume that the IUD does not alter HIV acquisition risk. Although non-hormonal, the effect of IUD-related inflammation is unknown; thus IUD use could affect HIV risk in either direction. Trials such as ours assess comparative risks between methods, not absolute risks. For example, similarity between methods could be due to no effect of either method, increased risk with both methods, or reduced risk with both methods. Comparative risks are of importance to women who desire contraception and wish to choose between alternative methods.

Keeping in mind the trial limitations, our findings suggest that any difference in the risk of HIV acquisition with IPC compared with the IUD is unlikely to be large. Some variation from the true effects in either direction may have been introduced by the loss to follow-up, method discontinuation and the use of NET in $16 \%$ of participants in the IPC arm. Most importantly, the study was not powered to detect a modest effect due to early closure of the trial. Therefore, we cannot rule out the possibility of an effect in either direction large enough to have important public health implications. An international Technical Consultation held at the WHO in Geneva on 31 January 2012 concluded that the WHO should continue to recommend that there are no restrictions on the use of hormonal contraceptive methods for women at high risk of HIV, due to insufficient evidence of an association. ${ }^{14}$ DMPA is a widely accepted and highly effective contraceptive method that is estimated to prevent thousands of maternal and infant deaths each year by reducing unintended pregnancy. ${ }^{15}$ A modelling of competing risks suggests that reducing DMPA use may only have public health benefits if the true HIV acquisition risk effect size approaches an odds ratio (OR) of 2.19. ${ }^{15}$ This modelling also showed that countries such as South Africa with high HIV incidence and high IPC use would see a reduction in total deaths with removal of IPC if there is a strong association between DMPA and HIV acquisition. ${ }^{15}$ However, for countries with high IPC use and high maternal mortality compared with HIV incidence, such as Bangladesh, Egypt and Indonesia, reducing IPC use would increase net deaths of women regardless of the OR estimate used. ${ }^{15}$ The net global effect of withdrawing IPC as a contraceptive option on deaths has been estimated to be 47000 fewer deaths per year with an OR of 2.19, 3400 more deaths with an OR of 1.2, and at least 16000 more deaths with an OR of 1.0, with a net increase in deaths in all countries. ${ }^{15}$

The strengths of our study include the potential for the findings to be generalisable to similar healthcare settings because the study took place within the routine health services, thus reflecting 'typical use' outcomes. The trial also addresses several factors which have a bearing on ongoing efforts to answer the HIV acquisition risk and hormonal contraception research question. First, it shows that many women without a specific preference for a contraceptive method were willing to agree to randomisation without any financial remuneration or other benefits. The acceptability and feasibility of randomisation is supported by the fact that discontinuation rates at final follow-up for both IPC and IUD were relatively low at a median follow up of 20 months. ${ }^{11}$

HIV prevention and contraception are two competing public health concerns and a framework for addressing the ethical challenges this poses for public health practice has been suggested. ${ }^{16}$ However, uncertainty about the relative effects of contraception on HIV may continue to undermine family planning and HIV prevention public health strategies, and impact 
service user confidence, without higher quality evidence. Data on the risk of HIV acquisition with other long-acting hormonal contraceptives such as implants are very limited, and it cannot be assumed that HIV acquisition risk will differ substantially between DMPA and these other contraceptive options without better evidence. Contraception providers should continue to counsel women on the measures available, including condom use and pre-exposure prophylaxis, to prevent HIV acquisition.

\section{CONCLUSIONS}

This study serves as a 'pilot' study to inform the feasibility and likely range of outcomes for a fully powered study. It is impossible to estimate the net effect of both known and unknown biological and behavioural adverse and protective effects of various contraceptive methods, including DMPA and other long-acting hormonal contraceptives, on HIV acquisition without more rigorous research. Our sub-study confirms the feasibility of randomised trial methodology to address this question. Robust evidence from larger RCTs of the relative effects of various contraceptive methods on HIV acquisition would provide a sound basis for global programmes to simultaneously promote safe contraception and reduce HIV incidence.

\section{Acknowledgements The costs of this study were internally funded by the Effective Care Research Unit (ECRU), East London, South Africa. The Eastern Cape Department of Health supplied the IPCs and IUDs provided to participating women at the study clinics. The authors gratefully acknowledge the women who supported the trial by way of participation, and staff at the Women's Health Clinics at Frere and Cecelia Makiwane Hospitals who contributed to the contraceptive care of women in the study. They acknowledge the contributions of Mrs Ana Nondlwana and other research staff of the Effective Care Research Unit.}

Contributors GJH contributed to study design, data analysis, and the drafting and revision of the report. MS contributed to study design, project management, and data collection. TAL contributed to data analysis and interpretation, and drafting and revision of the report. MT contributed to the drafting and revision of the report. EB contributed to study design. All authors reviewed and approved the final manuscript.

Funding Contraceptive care was provided by the routine clinical services and research costs were internally funded by the Effective Care Research Unit, East London, South Africa.

Competing interests GJH and MS are members of the Evidence for Contraceptive health and HIV Outcomes (ECHO) consortium that is undertaking a multicentre randomised trial of contraceptive methods and HIV acquisition.

\section{Patient consent Obtained.}

Ethics approval University of the Witwatersrand Committee for Research on Human Subjects, South Africa.

Provenance and peer review Not commissioned; externally peer reviewed.

Data sharing statement The authors support data sharing. Additional data on side effects of the contraceptive methods assessed in this study are still to be published.

Open Access This is an Open Access article distributed in accordance with the Creative Commons Attribution Non Commercial (CC BY-NC 4.0) license, which permits others to distribute, remix, adapt, build upon this work non- commercially, and license their derivative works on different terms, provided the original work is properly cited and the use is non-commercial. See: http://creativecommons.org/licenses/by$\mathrm{nc} / 4.0 /$

\section{REFERENCES}

1 Marx PA, Spira AI, Gettie A, et al. Progesterone implants enhance SIV vaginal transmission and early virus load. Nat Med 1996;2:1084-1089.

2 Trunova N, Tsai L, Tung S, et al. Progestin-based contraceptive suppresses cellular immune responses in SHIV-infected rhesus macaques. Virology 2006;352:169-177.

3 Quispe Calla NE, Vicetti Miguel RD, Boyaka PN, et al. Medroxyprogesterone acetate and levonorgestrel increase genital mucosal permeability and enhance susceptibility to genital herpes simplex virus type 2 infection. Mucosal Immunol 2016;9:1571-1583.

4 Blish CA, Baeten JM. Hormonal contraception and HIV-1 transmission. Am J Reprod Immunol 2011;65:302-307.

5 Roxby AC, Fredricks DN, Odem-Davis K, et al. Changes in vaginal microbiota and immune mediators in HIV-1-seronegative Kenyan women initiating depot medroxyprogesterone acetate. J Acquir Immune Defic Syndr 2016;71:359-366.

6 Fichorova RN, Chen PL, Morrison CS, et al. The contribution of cervicovaginal infections to the immunomodulatory effects of hormonal contraception. MBio 2015;6:e00221-15.

7 Murphy K, Irvin SC, Herold BC. Research gaps in defining the biological link between HIV risk and hormonal contraception. Am J Repro Immunology 2014;72:228-235.

8 Morrison CS, Chen PL, Kwok C, et al. Hormonal contraception and the risk of HIV acquisition: an individual participant data meta-analysis. PLoS Med 2015;12:e1001778.

9 Polis CB, Curtis KM, Hannaford PC, et al. An updated systematic review of epidemiological evidence on hormonal contraceptive methods and HIV acquisition in women. AIDS 2016;30:2665-2683.

10 Shisana O, Rehle T, Simbayi LC, et al. South Africa National HIV Prevalence, Incidence and Behaviour Survey, 2012. Cape Town, South Africa: HSRC Press, 2014.

11 Hofmeyr GJ, Singata-Madliki M, Lawrie TA, et al. Effects of the copper intrauterine device versus injectable progestin contraception on pregnancy rates and method discontinuation among women attending termination of pregnancy services in South Africa: a pragmatic randomised controlled trial. Reprod Health 2016;13:42.

12 Heffron R, Donnell D, Rees H, et al. Use of hormonal contraceptives and risk of HIV-1 transmission: a prospective cohort study. Lancet Infect Dis 2012;12:19-26.

13 Cates W, on behalf of the Evidence for Contraceptive Options and HIV Outcomes (ECHO) Consortium. Research on hormonal contraception and HIV. Lancet 2014;383:303-304.

14 World Health Organization. Hormonal Contraception and HIV: Technical Statement. Geneva, Switzerland, 2012. http://www. who.int/reproductivehealth/topics/family_planning/Hormonal_ contraception_and_HIV.pdf [accessed 10 August 2016].

15 Butler AR, Smith JA, Polis CB, et al. Modelling the global competing risks of a potential interaction between injectable hormonal contraception and HIV risk. AIDS 2013;27:105-113.

16 Haddad LB, Philpott-Jones S, Schonfeld T. Contraception and prevention of HIV transmission: a potential conflict of public health principles. J Fam Plann Reprod Health Care $2015 ; 41: 20-23$. 